\title{
Combining attributes in specified and categorized target search: Further evidence for strategic differences
}

\author{
SUSAN E. GATHERCOLE and DONALD E. BROADBENT \\ University of Oxford, Oxford, England
}

\begin{abstract}
Three experiments explored the processing of color and form. In these, subjects were required to report the color of a rectangle surrounding a target item that appeared in the middle of a stream of nontarget stimuli. A different pattern of errors was found when the target was cued by category rather than by identity, both when the target was a digit in a stream of letters (Experiment 1) and when it was a word in a sequence of words (Experiments 2 and 3). The results endorse those of McLean, Broadbent, and Broadbent (1982) by providing further convergent evidence that the processes involved in perceptual selection are not invariant, but instead can be characterized in terms of two alternative processing modes. In the first mode, the two task-relevant stimulus features are processed in parallel, whereas in the second mode, they are processed serially.
\end{abstract}

In experiments using a rapid sequential visual presentation (RSVP) technique, the task is to report a feature of a target item that appears in a series of briefly displayed visual events. For example, in the first reported RSVP study, by Lawrence (1971), the subjects were instructed to name the only word in a sequence of words that was printed in uppercase lettering. The target thus possesses two important features: the targetdefining attribute, which in Lawrence's case was the type of lettering, and the response attribute, which was the word itself. The principal concern of the present paper is to explore the mechanisms by which these two attributes of the target event are analyzed and combined in a RSVP task.

Two simple models of how the target response feature is correctly identified in this situation can be constructed. These are shown in Figure 1. It should be noted that, for simplicity, the figure assumes that the two stimulus attributes are fully processed by the time that the next item in the series arrives. This may not, of course, be the case. Fortunately, though, the predictions made on the basis of these two models are not materially affected by whether the two alternative modes of analysis operate on the external stimulus events or on an internal representation of them, such as a perceptual window that does not operate on a realtime basis.

According to the first, or "serial," model shown in Figure 1, each incoming stimulus is initially tested for

This research was funded by a grant awarded by the Science and Engineering Research Council to the first author. The second author is employed by the Medical Research Council. Requests for reprints should be sent to Susan E. Gathercole, Department of Experimental Psychology, University of Oxford, South Parks Road, Oxford, OX1 3UD, England.

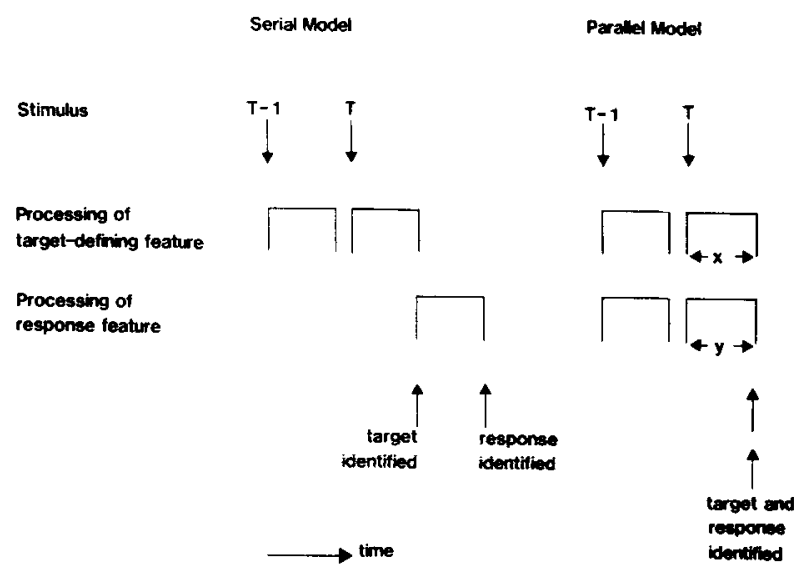

Figure 1. Serial and parallel models of processing the two task-relevant attributes of a stimulus in a RSVP task. The figure shows how, according to each model, the response attribute of the target $(T)$ is correctly identified. For explanations of intrusion errors, see text.

the presence of target-defining features. In the event of a positive identification's being made, analysis of the response attribute of the target will commence. When analysis is complete, the subject outputs the target response feature as the response. This serial processing model corresponds closely to the two-stage "detect and identify" notion proposed by Broadbent (1977). In contrast, according to the second, or "parallel," model, the two attributes of the target are analyzed simultaneously and independently. The response made by the subject corresponds simply to the response feature whose code is most active at the time at which the target-defining attribute is positively identified. This model has much in common with the views of Keele and Neill (1978) and Treisman (1977). 
One way in which the serial and parallel models can be distinguished empirically is by considering the errors made in these tasks. Lawrence (1971) noted that incorrect responses in RSVP experiments are most likely to originate from items appearing after the target in the list. The two models accommodate this phenomenon in different ways. According to the serial model, analysis of the response attribute of the target commences only after the target-defining attribute has been positively identified. Thus, if the response feature of a nontarget item is mistakenly analyzed, the item should be one that appears after, not before, the target in the series. When errors arise, they would thus be expected to originate in predominantly posttarget positions.

The account of these late intrusions provided by the parallel model depends on a particular assumption's being made about the relative processing times of the two stimulus attributes. This assumption is that it takes longer, on average, to identify the target-defining attribute than the response attribute of a stimulus. Thus, in Figure 1, time $\mathrm{x}$ has to be greater than time $\mathrm{y}$. Providing that this is the case, the response feature corresponding to a posttarget item may on occasion be most active at the time of target feature identification. Intrusion errors, therefore, should principally originate from items appearing after the target in the list.

The parallel account of posttarget intrusion errors is not, however, substantiated by recent results. The explanation hinges on the assumption that analysis of the target-defining attribute is more time-consuming than response feature analysis. By the same logic, if the task were reversed so that analysis of the target feature took less time than response feature analysis-in other words, if in Figure 1 time $x$ was less than time $y$-errors should principally originate from pretarget positions. McLean, Broadbent, and Broadbent (1982, Experiment 1) made this comparison and found that this was not the case. In their experiment, the RSVP lists contained colored letters, and the subjects' task was to identify either the color of a target letter or the letter printed in a target color. In both conditions, predominantly posttarget intrusions were found. These findings clearly favor the notion of conditional analysis of the response feature provided by the serial model.

Other data reported by McLean et al. (1982), though, cast doubt on an account of RSVP performance purely in terms of the serial model. On the basis of this model, it would be predicted that any factor that increased the time taken to test for the target-defining feature should lead to even later posttarget intrusions. In Experiment 2, each RSVP list contained a colored digit embedded in a list of colored letters. The task was to report the color of the digit. In the specified target condition, subjects were told the identity of the digit, and most errors corresponded to items that appeared later in the list than the target. In the categorized target condition, subjects did not now which digit the target would be. Given that it takes longer to categorize than to identify a digit (Dick,
1971; Posner, 1970; Taylor, 1978), the serial mode would predict even later intrusions in the categorizer than in the specified condition. In fact, errors wer found to be equally likely to originate from pre- an posttarget positions in the categorized case-that is, fron earlier in the list than in the specified condition, is which posttarget errors predominated.

In summary, neither of the two simple models con sidered here can successfully accommodate all of th results reviewed so far. The interpretation favored $b$ : McLean et al. (1982) is that these findings reflect th operation of two alternative strategies of processing, on corresponding to the serial model and the other to th parallel model. When subjects knew the identity of th target, as in Experiment 1 and in the specified conditios of Experiment 2, it was proposed that they adopted serial processing strategy. In other words, analysis of th response feature was suspended until the target had bees positively identified; thus, posttarget intrusions pre dominated. Performance in the categorized condition on the other hand, was explained in terms of a strateg. corresponding to the parallel model. It was assumed tha the analyses of the target and response attributes took on average, the same time-that is, in Figure 1, time : is equivalent to time $\mathrm{y}$-and that there was normal an independent variability in the time taken to encode th attributes. On this basis, symmetrical errors woul indeed be expected in this condition.

In view of the important implications that the result of McLean et al. (1982) appear to have for the role 0 : strategic modification of the processes involved in per. ceptual selection, three further experiments employin! the RSVP paradigm developed by McLean et al. werı conducted and are reported here. Experiment 1 explores the influence of spatial overlap of character and colo information on the differential pattern of errors ob tained in specified and categorized search conditions Experiments 2 and 3 compared specified and categorizer search in RSVP lists containing words.

\section{SPATIALLY SEPARATED COLOR AND CHARACTER INFORMATION}

The finding that errors originate earlier in a RSVI list when subjects search for the color of an unknowr rather than a known digit embedded in a list of letter: was explained by McLean et al. (1982) in terms of the operation of alternative strategies of perceptual process ing. It was suggested that parallel processing of color and characters characterizes search in the categorizec target condition, whereas a strategy of serial processing was favored in the specified target case. Given the counterintuitive nature of these results, though, it i: clearly important to consider any alternative and les: radical accounts of the data. One possibility is that color and characters were processed in parallel in both conditions in Experiment 2 of the McLean et al. paper, and 
that the differential pattern of errors merely reflected changes in the relative encoding times of the two attributes across the two conditions. The posttarget intrusions in the specified condition can be explained by longer encoding times for the target feature-the character-than for the response feature-the color. And searching for an unknown digit in the categorized condition may have slowed down color analysis to a greater extent than character analysis, such that the processing of the two attributes now took, on average, the same amount of time. Thus, errors originated equally often from before and after the target in this condition.

Why, though, should searching for an unknown rather than a known digit slow down color analysis? To answer this, it should be noted that in the McLean et al. (1982) study, the characters were printed in color, so that the color information and character information were completely overlapping in space. Thus, in the specified condition, the physical features of the target were known in advance. Because the rate of processing is known to be accelerated when the spatial location of target information is anticipated, it is possible that color analysis was facilitated at locations corresponding to target features in this condition. This speeded color analysis would not have been available in the categorized condition, in which the target features were not known in advance. For this reason, color processing may have been slower in the categorized than in the specified condition. It should be noted that we do not favor this nonstrategic account, because it fails to explain the results of the first experiment reported by McLean et al. As, however, it provides an explanation for the differential errors obtained in the two search conditions without recourse to strategies, it seems important to put this parallel account to the test. In Experiment 1 of the present paper, specified and categorized target search were compared in a RSVP task in which the color information and character information were spatially separated and the location of the color was always known in advance. If McLean et al.'s (Experiment 2) results are attributabie simply to a disruption in the speed of color processing in the categorized condition, they should not be replicated here.

In Experiment 1 of the present paper, each list contained letters and a digit, and the characters were printed in white on a black ground. Each character was surrounded by four colored lines that joined up to make a square. The square was always located in the same position, and the square and the character it bordered did not overlap. An example of a sequence of list items is shown in Figure 2. The subject's task was to identify the color of the square surrounding the digit. In further contrast to the McLean et al. (1982) study, in which the RSVP lists were constructed from a series of slides projected onto a screen, the experiments reported in this paper used computer-generated characters and colors displayed on the screen of a TV monitor.

\section{Experiment 1}

\section{Method}

Subjects. The Oxford Subject Panel provided 20 women, 18 to 45 years old, to participate in this experiment. All subjects were tested individually and were paid for their assistance.

Design. All subjects were tested in both the specified (S) and the categorized (C) target conditions. Each subject received the same sequence of 100 experimental lists, divided into four blocks each of 25 lists. All lists within a block were received in the same experimental condition. Ten subjects received the blocks in S-C-C-S order of conditions, and the other half received them in C-S-S-C sequence. Thus, over the 20 subjects, each list was received equally often in both experimental conditions. Three practice lists preceded the first block of lists, and two practice lists preceded the second block.

Apparatus. A Research Machines Limited $380 \mathrm{Z}$ microcomputer and keyboard were used to control presentation of the experimental stimuli. A Ferguson TX10 color monitor adapted to receive RGB input displayed the stimuli. The subjects were seated at a distance of approximately $1 \mathrm{~m}$ from the monitor.

Materials. The materials employed in this experiment followed the same principles of construction as those used in Experiment 2 of McLean et al. (1982). Of the 100 experimental lists employed in the present experiment, 50 lists used the same characters and color names as those employed in the McLean et al. (1982) experiment, and a further 50 were generated for the purposes of the present study.

Each list contained 16 letters and one digit, and every character was surrounded by a colored square, as shown in Figure 2. The characters were displayed in an area of the screen of width $.6 \mathrm{~cm}$ and height $.8 \mathrm{~cm}(.34 \times .46 \mathrm{deg}$ of visual angle $)$. The lines of the square were each of width $.2 \mathrm{~cm}$, and the outer edges of the square measured $1.5 \mathrm{~cm}$. Thus, all of the stimulus informa-

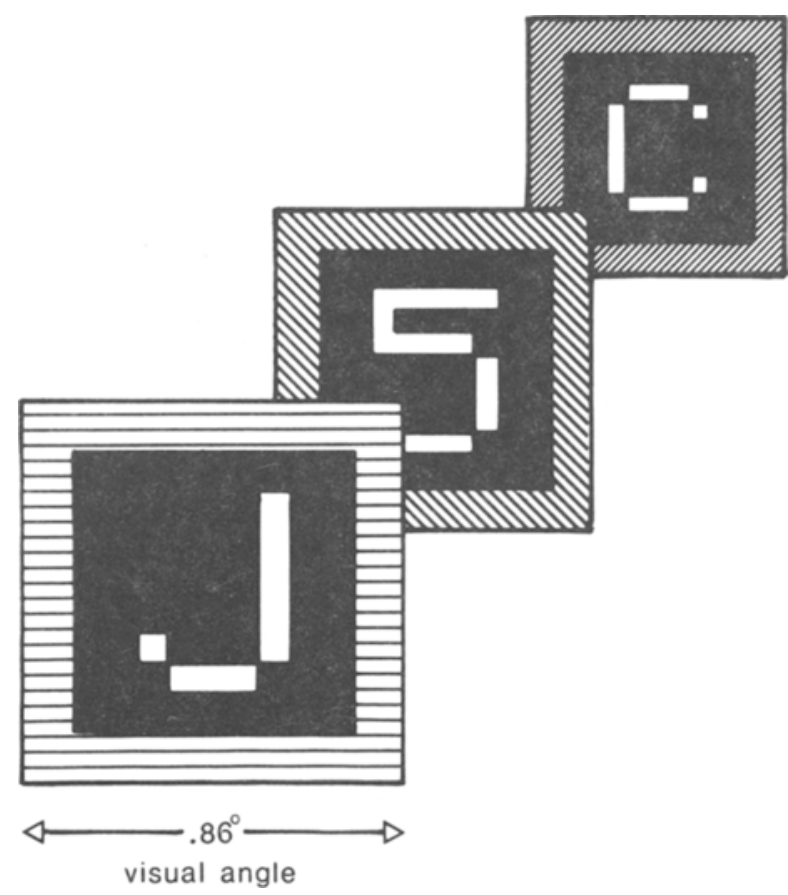

Figure 2. Three successive stimuli in the middle of a typical list in Experiment 1. The target stimulus, consisting of a digit surrounded by a colored square, is the second item in the figure. 
tion was contained in an area subtending $.86 \mathrm{deg}$ of visual angle in both width and height.

In each block of 25 lists, the target digit was located five times each at Positions 7, 8, 9, 10, and 11 in the 17-item list. The color of the target surrounding the digit was blue, green, red, yellow, or white. In order to maintain compatibility with the target character, which was the only digit in the list, the target color was not used at any other positions in the list. The digit was $3,4,5,6$, or 7 , and each of these targets was surrounded by a square of each color once in each block of 25 trials.

Consider now the 16 nontarget items in each list. The four letters occupying the two positions before and the two positions after the target were taken randomly and without replacement from the set $\mathrm{C}, \mathrm{F}, \mathrm{J}, \mathrm{N}$, and $\mathrm{X}$. Each of the four nontarget colors was used once at these positions. The first two and the last two letters in each list were taken from the set A, B, S, and $Z$, and were always surrounded by a purple square. The remaining eight letters in each list consisted of two each of the four letters that flanked the target. Two of each of the four nontarget colors were used for these items. The order of both these letters and colors was determined randomly, with the constraint that no two letters or colors were repeated at any successive positions in the list.

The target color was the only color in the list that appeared just once. Although no subjects spontaneously reported noting this, a consequence of this aspect of the design may have been to reduce errors by providing subjects with a clue to the correct response. There is, however, no reason to believe that this procedure could have led to any systematic bias in our results.

The five practice lists were constructed according to the same principles as the experimental lists.

Procedure. The subjects were seated in front of the color TV monitor and were familiarized with the nature of the experiment. A card listing the five experimental colors was placed in full view of the subjects, where it remained throughout the experimental session. At the beginning of each trial, the experimenter informed the subjects of the identity of the target in the forthcoming list. In the specified condition, the experimenter said aloud the digit, for example, "Three." In the categorized condition, the experimenter merely said, "Digit." The experimenter then initiated the presentation of the list. In both conditions, the subjects' task was to report the color of the square that accompanied the digit. The subjects were required to respond with the name of a target color at the end of every list, by guessing if necessary.

The stimulus list was displayed on the TV monitor. At the beginning of each list, a white fixation line appeared for $1 \mathrm{sec}$ in the center of the screen and was followed by a sequence of 17 items, each surrounded by a colored square. The character and color appeared simultaneously and stayed on the screen for approximately $80 \mathrm{msec}$, when they were replaced by the next item and color. At the end of the list, the screen cleared. The subjects were then required to provide their response, which the experimenter recorded before initiating the next trial. Rest pauses were given at the end of each of the four blocks of trials, and the experimental session for each subject lasted about $20 \mathrm{~min}$.

The first practice list in the experiment was used to demonstrate the procedure. In the remaining two practice trials given before the first block of lists, and those given before the second block, the experimenter provided the subjects with feedback concerning the accuracy of their responses. No such information was provided during the experimental trials.

\section{Results}

The data were scored by assigning all responses to one of the five categories corresponding to the list positions at which the reported color appeared. These ranged from two positions before the target $(-2)$ to two positions after the target $(+2)$. Correct responses were categorized as $\mathrm{T}$, the target position. Table 1 shows the results.

Despite the close temporal proximity of the colors of successive stimuli in this experiment, performance in both conditions was well above the $20 \%$ correct level that would be expected on the basis of chance responding. More intrusions were found at posttarget than at pretarget positions in both experimental conditions. Sign tests performed on the data showed a posttarget dominance of errors, both when only target-adjacent positions were compared $(p<.001$ and $p<.01$ in the specified and categorized conditions, respectively) and when Positions 1 and 2 on both sides were included $(p<.05$ in both conditions).

The principal comparison of interest across the specified and categorized conditions is the relative frequency of posttarget to pretarget error responses. It appears from Table 1 that there was a greater proportion of posttarget than of pretarget errors in the specified than in the categorized target condition. But because the numbers of errors made in the two conditions were not equivalent, direct comparisons of the frequency of errors at each position could not be made across the two conditions. The ratio of posttarget to pretarget responses was therefore used as the measure for comparison across the two conditions. At targetadjacent positions, the ratios were 2.01 in the specified condition and 1.46 in the categorized condition; when Positions 1 and 2 on both sides of the target were taken into account, the ratios were 1.81 and 1.25 , respectively. For the purposes of statistical analysis of these data, the two post- and pretarget intrusion ratios were calculated for each subject, and an analysis of variance was performed on each set of scores. It was found that the ratios of post- to pretarget responses were indeed significantly greater in the specified than in the categorized target condition, both when the target-adjacent positions were tested $[F(1,19)=4.57, p<.05]$ and when the two positions on each side of the target were included in the ratio $[F(1,19)=7.40, p<.05]$.

Thus, the result of McLean et al. (1982) has been confirmed with different color information and different apparatus, and with a solely female population rather than with a group of subjects of both sexes. Once again, posttarget intrusion errors were relatively more frequent than pretarget intrusion errors in the specified target than in the categorized target condition.

Table 1

Mean Percent Responses Originating at Each of the Five Possible List Positions in the Specified and Categorized Target Conditions in Experiment 1

\begin{tabular}{lrrcrrr}
\hline & \multicolumn{5}{c}{ List Position } \\
\cline { 2 - 6 } & -2 & -1 & T & +1 & +2 \\
\hline Specified Digit & 5.9 & 12.3 & 48.8 & 24.7 & 8.3 \\
Categorized Digit & 11.7 & 14.7 & 40.7 & 21.4 & 11.6 \\
\hline
\end{tabular}


Two further features of the data are noteworthy. First, although more correct responses were given in the specified than in the categorized condition $(48.8 \%$ as opposed to $40.7 \%$ ), the difference was not significant by sign test $(p>.05)$. And second, more error responses were made at target-adjacent positions $( \pm 1)$ than at \pm 2 positions $(\mathrm{p}<.05$ in both conditions). This immediacy effect was also noted in the corresponding experiment in the McLean et al. (1982) study, and is of considerable importance because it establishes that in both conditions the error responses were not merely random guesses, but were informative failures of the system.

\section{Discussion}

The distribution of errors obtained in a task in which subjects had to identify the color of a digit embedded in a list of letters was influenced by the type of search information provided. A greater predominance of errors corresponding to items appearing after the target in the RSVP list was found when subjects knew in advance the identity of the target digit than when they did not. These results replicate those of McLean et al. (1982), and generalize them to a procedure in which the colors and characters were spatially separate rather than completely overlapping.

The finding that spatial overlap of colors and characters was not critical to the differential pattern of errors obtained in specified and categorized search rules out one account of the McLean et al. (1982, Experiment 2) results considered earlier in this paper. According to that account, analysis of color and of characters proceeds in parallel, and the earlier errors obtained in categorized than in specified search are attributable to impaired color processing when the location of the target cannot be anticipated. In the present experiment, however, the location of the color was completely predictable and independent of the target, so that knowledge of target identity would not have influenced speed of color analysis. These results thus lend further weight to McLean et al.'s proposal that the more symmetrical pattern of errors obtained in categorized than in specified target search reflects the adoption of a parallel rather than of a serial mode of color and character analysis.

\section{SPECIFIED AND CATEGORIZED WORDS IN RSVP}

The phenomenon of central interest to this paperthe systematic differences in errors obtained in a RSVP task when targets are specifically and categorically defined-has so far been found with colored characters (McLean et al., 1982) and white characters surrounded by colored squares (Experiment 1 in this paper). In both cases, the RSVP lists consisted of alphanumeric stimuli, and the subjects' task was to identify the color presented with the digit embedded in a list of letters. On the basis of our results, we have suggested that when only the category of the target is known, subjects tend to process the character and color simultaneously. In contrast, it appears that a more selective processing strategy may be adopted when subjects know the identity of the target. This strategy is one of initially testing the character for evidence of target-defining features, and analyzing color only if such features are positively identified.

It may, however, be the case that the option of parallel attribute processing is available only when the task involves categorical selection among alphanumeric stimuli. Letters and digits are both small and extremely well-learned categories known to possess some unique psychological features (e.g., Brand, 1971; Rabbitt, 1978). Furthermore, each alphanumeric stimulus conveys much less information (in information theory terms) than any word. It is therefore conceivable that in our experiments analysis of color can proceed in parallel with a series of tests designed to distinguish digits from letters only because of either the highly learned nature of these operations or the relatively minor informational load these stimuli impose on the processing system. If this is the case, the search through a list for a word defined by its membership in a semantic category may, as a result of requiring more elaborate analysis, preclude parallel processing of color.

Two further experiments were therefore conducted in order to determine whether a search for the color of a square surrounding specified and categorized words in our RSVP task would yield the differential pattern of intrusion errors we have shown to characterize task performance when alphanumeric stimuli are employed. If, for the reasons outlined above, the categorical search for a digit among letters is a unique case that promotes parallel processing, no difference in the distribution of errors around the target would be expected in the specified and categorized word conditions. If, however, the differential pattern of errors obtained in earlier experiments reflects the operation of fundamentally different strategies of perceptual selection, the greater preponderance of errors arising from post- than pretarget positions with specified than with categorized search information should be obtained as readily with words as with letters and digits.

Experiment 2 was designed to correspond as closely as possible to Experiment 1, except that the list items were words rather than alphanumeric characters. Each list consisted of 17 four-letter words enclosed by a nonoverlapping colored rectangle. The subjects' task was to report the color of the rectangle surrounding the target word, which was identified either specifically or by semantic category. Thus, if, for example, the target word was "GOLD," in the first condition the experimenter would say, "GOLD," whereas in the categorized condition the subjects would be told, "A metal" before the list was displayed.

The target words employed in Experiment 2 came from 50 different semantic categories. This large number of classes was used in order to minimize the likelihood 
that cognitive operations designed to test for the presence of target category features would, over the course of the experiment, become well-learned or automatized.

\section{Experiment 2}

\section{Method}

Subjects. Twenty women from the Oxford Subject Panel, 18 to 45 years old, participated in the experiment. All subjects were tested individually and were paid for their assistance.

Design and Apparatus. These were identical to those used in Experiment 1.

Materials. To as great an extent as possible, the materials were constructed according to the same principles employed in Experiment 1. Again, the RSVP lists consisted of 17 items, each surrounded by four colored lines joined together to form a rectangle. In Experiment 2, however, the list items were fourletter words rather than single alphanumeric characters. The words, when displayed on the TV monitor, were of width $3.0 \mathrm{~cm}$ and height $.8 \mathrm{~cm}(1.72 \times .46 \mathrm{deg}$ of visual angle), and the outer edge of the rectangle measured $3.9 \times 1.5 \mathrm{~cm}(2.24 \times .86 \mathrm{deg}$ of visual angle).

The target word appeared five times each at Positions 7, 8, 9,10 , and 11 in each block of 25 lists. All of the targets were nouns taken from one of the nine most popular category exemplars generated for a given category according to Battig and Montague (1969). Two exemplars from each of 50 categories were selected for use in this experiment. The two exemplars from each category were included in either the first and third blocks of lists or the second and fourth blocks. Thus, each category was represented once in the first half of the experimental session and once in the second half. The first 2 and last 2 words in each list were taken randomly and without replacement from the set "RENT," "TANK," "ACID," and "PEST." The remaining 12 words in each list were taken from a population of 300 nouns. The target words were grouped into four frequency bands according to the corpus frequency count of Kucera and Francis (1967), and target and filler words in each list were matched within a band.

The sequence of colors enclosing the words in each list was identical to that used in Experiment 1, except that the color orange was substituted for white, which had proved to be less discriminable than the four other colors in the set-blue, green, red, and yellow.

Procedure. The procedure differed from that followed in Experiment 1 in only one respect, and this concerned the exposure time of the list items. Each word and its bordering color rectangle were displayed for approximately $200 \mathrm{msec}$. This display duration was adopted on the basis of pilot investigations that had established that it would produce performance levels comparable to those obtained in Experiment 1.

\section{Results}

All responses were assigned to one of five categories, according to the position in the list at which the reported color appeared. The experimental sequence positions ranged from -2 (two positions before the target in the list) to +2 (two positions after the target). Correct responses were categorized as $\mathrm{T}$, target position. The results are shown in Table 2.

It appears from Table 2 that the number of error responses that originated at list positions before and after the target was more or less equal in the categorized target condition, but asymmetric in the specified case, in which a clear majority of intrusions occurred at post-
Table 2

Mean Percent Responses Originating at Each of the Five Possible List Positions in the Specified and Categorized Target Conditions in Experiment 2

\begin{tabular}{lrrrrr}
\hline & \multicolumn{5}{c}{ List Position } \\
\cline { 2 - 6 } & -2 & \multicolumn{1}{c}{-1} & \multicolumn{1}{c}{ T } & +1 & +2 \\
\hline Specified Word & 5.6 & 6.0 & 72.7 & 11.0 & 5.4 \\
Categorized Word & 2.2 & 15.2 & 44.9 & 16.6 & 10.1 \\
\hline
\end{tabular}

target positions. Sign tests confirmed that this was the case by showing that at both target-adjacent positions, and Positions 1 and 2 on either side of the target, there were more post- than pretarget errors in the specified condition $(\mathrm{p}<.05)$ but not in the categorized condition $(p>.05)$. This pattern of results is reflected in the ratios of posttarget to pretarget errors, which in the specified and categorized conditions are 1.83 and 1.09 , respectively, when the target-adjacent positions are tested, and 1.41 and 0.97 , respectively, when the two list positions on each side of the target are included in the measure. Ratios of the posttarget to pretarget intrusions for each subject were calculated according to both measures, and two analyses of variance were performed on the scores. The effect of experimental condition on post- and pretarget error ratios was significant for both the one-position scores $[\mathrm{F}(1,19)=5.01, \mathrm{p}<.05]$ and the two-position scores $[F(1,19)=4.49, p<.05]$. In summary, specific target information yielded a relatively greater frequency of post- than of pretarget errors than did target category information.

It is apparent from Table 2 that performance was considerably worse on this task when the subjects were provided with the semantic category of the target as search information $(44.9 \%$ correct) than when the specific word was given $(72.7 \%)$ correct). The difference was found to be highly significant by sign test $(p<.001)$. However, there is some evidence from the data that the subjects were not responding merely randomly in the error trials in the categorized condition. In this case, as well as in the specified target condition, more intrusion errors were found at Position +1 than at Position +2 ( $p<.001$ in both cases, by sign test). This suggests that at least a sizable proportion of the errors were misperceptions and not pure guesses.

\section{Discussion}

Providing the subjects with information concerning the semantic category of a target word in a RSVP task yielded a pattern of errors different from that yielded when the identity of the target word was known. In the specified target case, a relatively greater number of intrusions came from posttarget than from pretarget positions than in the categorized condition. It therefore now appears that the more symmetrical pattern of errors obtained in Experiment 1 and McLean et al. (1982, Experiment 2) following categorized target search, and 
believed to reflect parallel processing of color and form information, is not dependent on unique properties of alphanumeric stimuli. Instead, it seems that, irrespective of the target and nontarget categories used, alternative processing strategies are available to the individual in a task of this kind.

These latest results considerably strengthen the case for arguing for a theoretical framework that accommodates flexibility and not invariance in processing mechanisms. Our findings suggest that subjects may be more likely to test for the presence of the target before initiating color processing if they know the identity of the target. When the category of the target is the only search information, on the other hand, there seems to be a greater tendency for subjects to adopt a strategy of processing the form and color attributes simultaneously. Moreover, the availability of this second mode does not seem to depend on using small and well-learned search categories such as digits and letters.

One potential interpretational difficulty with the results of Experiment 2 should, however, be acknowledged. This concerns the higher level of performance attained in the specified than in the categorized word condition. It is possible that the symmetrical pattern of errors obtained in the categorized condition in Experiment 2 was a consequence of increased task difficulty. Specifically, the subjects may have made more pure guesses concerning target color in this condition than in the specified case, and this may have masked a residual pattern of predominantly posttarget intrusions in the categorized condition.

Because this ambiguity in the results of Experiment 2 considerably weakened our argument for the generality of the alternative processing strategies available in this search task, a further experiment, in which words were once again employed as the list stimuli, was designed. The principal difference between the two experiments was that, in Experiment 3, performance level in the specified and categorized target conditions was equated. This was achieved by imposing a random dot matrix onto the area of the TV screen occupied by the list words in the specified condition. By degrading the quality of the visual display in this condition, performance was reduced to the level reached in the categorized condition, in which the visual display remained free of visual noise.

Some characteristics of the list materials were also changed in Experiment 3. In Experiment 2, the target words were popular category exemplars, and target category always changed over successive lists. It was suspected that one consequence of these aspects of the design may have been to have encouraged subjects to formulate a hypothesis concerning target identity in the categorized condition, which would to some extent have equated the two experimental conditions. In Experiment 3, the target words were designed to be less predictable in order to make such a guessing strategy less useful. Relatively infrequent category exemplars were employed as targets, and five such instances of each category were used as targets in successive lists. The purpose of Experiment 3 was therefore both to test the guessing hypothesis and to encourage the adoption of different processing strategies across the specified and categorized target conditions.

\section{Experiment 3}

\section{Method}

Subjects. The Oxford Subject Panel provided 20 women, between 18 and 45 years old, none of whom had been tested in Experiment 2, to participate in this experiment. All subjects were tested individually and were paid for their services.

Design and Apparatus. The same design and equipment used in the two previous experiments were employed in this experiment.

Materials. As in Experiment 2, each of the 100 experimental lists consisted of 17 words, each surrounded by a nonoverlapping colored rectangle. All lists contained five-letter words. Each measured width $3.8 \mathrm{~cm}$ and height $.8 \mathrm{~cm}(2.18 \times .46 \mathrm{deg}$ of visual angle) when displayed on the TV screen, and the outer edge of the colored rectangle measured $4.7 \times 1.7 \mathrm{~cm}(2.7 \times$ $.98 \mathrm{deg}$ of visual angle).

The first and last 2 words in each list were taken randomly and without replacement from the set "TOTAL," "CLASS," "PLANE," and "STAFF." In each block of 25 lists, the target word was located five times each at Positions $7,8,9,10$, and 11 . The 100 target words consisted of five exemplars from each of 20 categories selected from Battig and Montague (1969). The targets were all words of low frequency of generation in their category $\rightarrow$ on average, they were the 20 th most popular category instances, in contrast to the targets in Experiment 3, which were, on average, the 3 rd most popular category instances. In Experiment 3 , in all but one category, the targets were nouns. In the remaining category, that of color, the exemplars were adjectives. The 12 remaining items in each list were taken from a population of 600 nouns. The target words were grouped into four frequency bands according to Kucera and Francis (1967), and target and nontarget words in each list were matched for frequency band. Each nontarget word appeared in the lists, once in each of the first two and second two blocks of lists.

The sequence of colors of the rectangles enclosing the list items was identical to that used in Experiment 2.

Over two groups of 10 subjects, the same lists were received equally often in the specified and categorized target conditions, but one aspect of the visual display did differ in the two cases. In both conditions, each list word was printed in white on a black TV screen and enclosed in a colored rectangle. In the specified target condition, however, a white dot matrix was superimposed on the area of the screen also occupied by the word. A grid of small dots was constructed to cover the word area (but not the colored area), and $20 \%$ of the dots were colored white rather than black. The matrix was generated randomly by the computer for each subject at the beginning of each block of specified target trials. The $20 \%$ density of noise was chosen as a result of pilot investigations that had established that this degree of degradation was sufficient to reduce performance in this condition to the level achieved in the noise-free categorized target condition.

Procedure. In most respects, the same general procedure was followed in this experiment as in Experiment 2. Due to the use of longer words as list items than in Experiment 2, the exposure duration of each list item was increased to approximately $260 \mathrm{msec}$ in order to produce equivalent levels of performance in Experiments 2 and 3. 
The subjects were informed that the visual display would be degraded in the specified condition and were told that the white dots were unimportant and should be ignored.

\section{Results}

Responses in the specified and categorized target conditions were classified according to the experimental list position at which the reported color occurred.

It is clear from Table 3 that the procedure of impos. ing a random dot matrix onto the list words in the specified target condition was successful in reducing performance to a level equivalent to that reached in the categorized condition ( $47.2 \%$ and $44.9 \%$, respectively). A sign test confirmed that the small difference between the two conditions was not significant $(\mathrm{p}<.05)$.

With respect to the relative frequency of responses originating at positions before and after the target, the results appear to correspond well to those obtained in Experiment 2. Predominantly posttarget intrusions were found in the specified condition but not in the categorized condition. Sign tests on two measures, comparing the number of errors at target-adjacent positions and at both positions on each side of the target confirmed this observation $(p<.05$ and $p<.005$ in the specified condition with the one-position and two-position measures, respectively; $\mathrm{p}>.05$ in the categorized condition, in both cases).

Once again, the ratio of post- to pretarget intrusions was greater in the specified than in the categorized target condition, both when the Position 1 measure was taken (1.71 and 1.23 , respectively) and when both positions were included (1.53 and 1.14, respectively). However, the analysis of variance performed on the ratios calculated for each subject failed to establish this difference as significant. The effect of condition on posttarget to pretarget positions was nonsignificant, both when target-adjacent positions were considered $[F(1,19)=$ $1.23, \mathrm{p}>.05]$ and when all intrusions were included $[F(1,19)=1.14, p>.05]$.

These results are rather puzzling. Although from the mean data it looks as though the same pattern of greater dominance of post- than of pretarget intrusions in the specified than in the categorized word conditions is present in this experiment as in Experiment 2, in statistical terms the difference is not reliable. This situation clearly makes it difficult to draw any strong conclusions on the basis of these data. In order to resolve this ambiguity, the data from Experiments 2 and 3 were combined, and the difference between the experiments

Table 3

Mean Percent Responses Originating at Each of the Five Possible List Positions in the Specified and Categorized Word Target Conditions in Experiment 3

\begin{tabular}{lrcccc}
\hline & \multicolumn{5}{c}{ List Position } \\
\cline { 2 - 6 } & \multicolumn{1}{c}{-2} & -1 & T & +1 & +2 \\
\hline Specified Word & 9.1 & 11.8 & 47.2 & 20.2 & 11.7 \\
Categorized Word & 11.7 & 14.1 & 44.9 & 17.4 & 11.9 \\
\hline
\end{tabular}

was treated as a between-subjects factor within a single analysis of variance. Two such analyses were performed, one for target-adjacent positions and one for all intru. sions. In the analysis of target-adjacent ratios, there was a significant main effect of condition $[F(1,38)=6.23$, $\mathrm{p}<.05]$, but neither the experiment term $[\mathrm{F}(1,38)<$ 1] nor the interaction between condition and experiment $[F(1,38)=2.52, p>.05]$ reached significance. The same pattern of outcomes emerged from the analysis of the two-position measure of post- to pretarget intrusions. The effect of condition was significant $[F(1,38)=5.48, p<.05]$, and neither the experiment effect $[F(1,38)<1]$ nor the interaction between condition and experiment $[F(1,38)=1.09, p>.05]$ was significant. These outcomes establish that there were no significant differences between the patterns of results obtained in Experiments 2 and 3. On this basis, it seems justifiable to conclude that although the differences in the characteristics of the errors obtained in the specified and categorized word conditions in Experiment 3 were not as great as in Experiment 2, the two experimental conditions influenced performance in a corresponding manner in the two cases.

One final aspect of these results deserves comment. As in Experiments 1 and 2, more intrusion errors were obtained in both experimental conditions at targetadjacent positions than at Positions \pm 2 (by sign test, $p<.05$ in the specified case and $p<.005$ in the categorized case).

\section{Discussion}

The results of Experiment 3 show a pattern of differential intrusion errors across the specified and categorized conditions in this RSVP task that corresponds closely to the pattern obtained in Experiment 2. Specified word targets led to mainly posttarget errors, whereas categorized targets yielded almost as many errors that originated from pretarget as from posttarget positions. Moreover, in Experiment 3 , equivalent levels of performance were reached in the specified and categorized target conditions, as a result of the procedure of degrading the word information in the specified target case. This result suggests that the more symmetrical distribution of errors around target position in categorized search in Experiment 2 was not due simply to the task being more difficult in this condition. The possibility that random responding occurs with different frequencies in the two conditions in Experiment 3 cannot, of course, be ruled out. These findings do, however, converge with the results of the other experiments reported in this paper in suggesting that the differential distribution of errors obtained in specified and categorized target search is a genuine and generalizable phenomenon.

\section{GENERAL DISCUSSION}

The findings of the three experiments reported in this paper fit well with the view put forward by McLean 
et al. (1982) that the way in which stimulus events are processed depends on the type of the search information available to the subject. The McLean et al. study established that in a RSVP task in which the subjects have to report the color of a target, errors originate mainly from list positions after the target when the target is specified. In contrast, when only the category of the target is known, the errors arise equally frequently from before and from after the target. This finding has now been established to be both reliable and generalizable. Experiment 1 in this paper, in which each RSVP list consisted of letters and a digit surrounded by nonoverlap. ping colored squares, determined that this phenomenon was not dependent on using stimuli in which the color and form dimensions overlapped in location. Experiments 2 and 3 demonstrated that the differential patterns of errors found in the specified and categorized search conditions are not restricted to search among constrained and well-learned search categories such as digits and letters. The same results were obtained when word lists were used and the target was specified either by its identity or its membership of a semantic category.

Two alternative models of performance in a RSVP task were contrasted at the beginning of this paper. The first was a serial model, which characterizes processing as involving initial analysis of the target-defining attribute of each stimulus, analysis of the response attribute being conditional on a positive outcome to this test (Broadbent, 1977). The parallel model, in contrast, assumes simultaneous processing of the two stimulus attributes and is closely related to the views of Keele and Neill (1978) and Treisman (1977).

We believe that the results of the experiments reported here, and the earlier findings of McLean et al. (1982), argue against both of these simple models. Instead, the data suggest that two alternative modes of processing are available and that these are differentially favored by search condition. When the identity of a target is known, a strategy corresponding to the serial mode appears to be adopted-the predominance of posttarget errors suggests that color processing is not initiated until evidence for target presence is detected. Such a strategy might be expected to be effective in this situation, because knowledge of target identity should enable speedy interrogation for target features. In contrast, when only the category of the target is known, performance would be likely to be maximized by the adoption of a strategy corresponding to the parallel model, which would in volve continuous processing of both color and form information. The more symmetrical errors obtained in categorized than in specified search are certainly consistent with such a parallel mode of analysis.
The experiments reported in this paper explore the processes involved in the perceptual selection of color and form information. The evidence that has been collected so far suggests that color and form can be analyzed either in parallel or in sequence, and that these alternative strategies of processing are available when the search items are words as well as when they are letters and digits, and with spatially separated as well as overlapping color and form information. Several issues that have not been addressed so far, though, certainly merit consideration. One particularly important question is whether the availability of these processing strategies is restricted to pairs of features, such as form and color, that might be expected to be represented in different cognitive channels or whether they extend to other attribute combinations, such as two verbal items.

\section{REFERENCES}

Battig, W. F., \& Montague, W. E. (1969). Category norms for verbal items in 56 categories: A replication and extension of the Connecticut category norms. Journal of Experimental Psychology Monographs, 80(3, Pt. 2).

Brand, J. (1971). Classification without identification in visual search. Quarterly Journal of Experimental Psychology, 23, 178-186.

Broadbent, D. E. (1977). The hidden preattentive processes. American Psychologist, 32, 109-118.

Dick, A. O. (1971). Processing time for naming and categorization of letters and numbers. Perception \& Psychophysics, 9, 350-352.

KeEle, S. W., \& Neill, W. T. (1978). Mechanisms of attention. In E. C. Carterette \& M. P. Friedman (Eds.), Handbook of perception (Vol. 9). New York: Academic Press.

KuĆERA, H., \& Francis, W. N. (1967). Computational analysis of present-day American English. Providence, RI: Brown University Press.

LAWRENCE, D. H. (1971). Two studies of visual search for word targets with controlled rates of presentation. Perception \& Psychophysics, 10, 85-89.

McLean, J. P., Broadbent, D. E., \& Broadbent, M. H. P. (1982). Combining attributes in rapid sequential visual presentation tasks. Quarterly Journal of Psychology, 35A, 171-186.

Posner, M. I. (1970). On the relationship between letter names and superordinate categories. Quarterly Journal of Psychology, 22, 279-287.

RABBidT, P. (1978). Sorting, categorization and visual search. In E. C. Carterette \& M. P. Friedman (Eds.), Handbook of perception (Vol, 9). New York: Academic Press.

TAYLOR, D. A. (1978). Identification and categorization of letters and digits. Journal of Experimental Psychology: Human Perception and Performance, 4, 423-439.

Treisman, A. M. (1977). Focused attention in the perception and retrieval of multidimensional stimuli. Perception \& Psychophysics, 22, 1-11.

(Manuscript received November 11, 1983; revision accepted for publication April 18, 1984.) 